\title{
Clinical Audit of Two Standard Surgical Site Treatments Following Dental Extractions and The Impact on Incidence of Alveolar Osteitis Within a Specialist Dental Clinic
}

Myles Dakin ( $\square$ myles@specialistdentalpartners.co.uk)

Specialist Dental Partners, Unit B9, Melbourn Science Park

Richard Aspinall

Coventry University

Thomas Kenny

Hypo-Stream Ltd, 31A Charnham Street, Hungerford

\section{Research Article}

Keywords: Surgical site, aqueous chlorine in physiological saline, alveolar osteitis

Posted Date: December 14th, 2021

DOI: https://doi.org/10.21203/rs.3.rs-1159818/v1

License: (c) (1) This work is licensed under a Creative Commons Attribution 4.0 International License.

Read Full License 


\section{Abstract \\ Background}

Alveolar osteitis is a painful condition following dental extraction associated with absence of blood clot and consequent exposure of underlying alveolar bone. It is a condition whose exact aetiology is unresolved and a known complication in up to $30-40 \%$ of dental extractions. The following report details a retrospective evaluation of two approved, long-standing wound care treatments used, post-extraction, within our clinic to assess for relationship with alveolar osteitis incidence.

\section{Methods}

Patients undergoing non-acute dental extraction at a dental out-patient clinic in the UK had received two different standard treatments for a period from 2000 to 2014. The treatments were mouth rinse with physiologic saline or surgical site irrigation and mouth rinse with physiologic saline-low dose aqueous chlorine used in wound and burn-site care. Retrospective, post-operative outcome analysis suggested improved outcomes with one of the standard treatments versus the other. A full retrospective audit of consecutive treatment outcomes was implemented to test the hypothesis. The audit data-collection was blinded to the treatment administered and to the identity of the treating clinician. Statistical analysis was carried out independently and blinded to the treatment modality.

\section{Results}

All patients were routinely reviewed within seven days and a determination of un-complicated healing vs alveolar osteitis (requiring further intervention) was made. Our results show that treatment with of aqueous chlorine in physiological saline significantly reduced the incidence of alveolar osteitis versus the control group who received physiologic saline without socket irrigation and chlorhexidine oral rinse homecare. (Odds Ratio 0.0146 Relative Risk $0.029(95 \% \mathrm{Cl} 0.0093$ to 0.0928$)$ significance $(p<0.001)$.

\section{Conclusions}

This is the first report to show an extremely significant reduction in the incidence of alveolar osteitis following treatment with low dose aqueous chlorine in physiological saline compared with those receiving the physiologic saline and saline mouth rinse or Corsodyl mouth rinse. This shows an approach to surgical site care is superior to the most common, reported, standard of care treatment. The impact of this has led to improved antibiotic stewardship within the clinic for dental extraction or elective oral surgery, including third molars.

\section{Background}


Very low dose aqueous chlorine solutions have longstanding use in clinical wound care; most commonly burns, non-healing venous ulcers and diabetic foot ulcers. Low dose hypochlorite formulations are approved by both the European Medicines Agency (EU - Amukine Med Amuchina S.r.l. Via Pontasso, 13 - 16015 Casella [Genova], Italy: AIC n. 032192015) and Food \& Drug Administration (USA - Dakin's Solution Century Pharmaceuticals Inc. 10377 Hague Road, Indianapolis IN 46256 USA) for use as over the counter non-prescription products as topical disinfectants in wound care. Several reports of clinical use of low concentration aqueous chlorines are published in peer reviewed journals and specifically addressing the potential to disinfect contaminated surgical or wound sites without producing adverse effects $[12,13,14,16]$.

The clinical use of very low level hypochlorite has been extensively reported for over 70 years $[12,13,14$, 16]. In addition to the long standing evidence of clinical utility there are multiple reports and reviews of pre-clinical investigations which address safe concentration and efficacy as an antimicrobial $[7,12,16]$. Furthermore, the endogenous production of hypochlorite by neutrophils as a derivative of myeloperoxidase produced hypochlorous acid is described by investigators of neutrophil function [15, 17]. Indeed, a review of the biochemistry of endogenous myeloperoxidase function calculates a highest level of concentration of the endogenous hypochlorous acid-hypochlorite blend in inflammatory foci as between 25-50nM (equivalent to $1300-2600$ ppm) [15]. The same review of the function of this endogenously produced very low dose hypochlorous acid-hypochlorite compound blend examines the reactions of the compounds with multiple key biologically important structural and messenger molecules and compounds including proteins, fatty acids, cholesterol-derived hormones and nucleic acids [15]. The implication of these analyses suggests that a concentration and formulation close to that found endogenously can be produced by a dilution of approved formulations. If such a biomimetic formulation were produced it should be possible to maintain wound or surgical sites with a low level of microbial contamination without resorting to antibiotics. Within this clinical evaluation, the formulation of the combination of hypochlorite, hypochlorous acid and saline was used as a surgical site and wound wash and this was derived from approved compounds.

One of the most common and painful conditions following dental extraction is alveolar osteitis or "dry socket" which is often linked to repeated visits to the dental surgery or the hospital and an increased burden, financially, physically and psychologically on the patient.

As a result this condition has been linked with a number of contributing factors and so consequently a large range of treatments are offered including rinsing of the socket with

chlorhexidine or saline or placement of specific dressing. Whilst true infection and osteomyelitis is rare following dental extraction and oral surgery, alveolar osteitis has been reported as a known complication following as many as $30-40 \%$ elective oral surgical procedures [1-5]. Antibiotic use following dental extraction is common. In 2012 both Tolstunov [4] and Yengopal [5] reported on alveolar osteitis and current methods used to reduce its incidence. Neither found evidence of effectiveness using chlorhexidine 
$(0.2 \%$ solution or gel) or isotonic (normal) saline surgical site irrigation [4, 5]. Indeed, the most startling finding was that doing nothing was preferable to using any surgical site treatment [4].

In a recent investigation of a concentrated growth factor administered to dental extraction surgical sites reported a reduction in the incidence of alveolar osteitis versus a control group, where antibiotics were also routinely administered prospectively [6].

However there have been growing concerns about the increased use of antibiotics and with this in mind we were aware that component oxidizing agents present in unbuffered dilute sodium hypochlorite in saline solutions have previously been shown to have antibacterial properties [7]. Such approaches have been used over many years principally in the field of burns care and in the care of gunshot trauma and treatment of non-healing leg ulcers $[7,13,14]$. Moreover such solutions have also been suggested to assist wound repair through their ability to induce the proliferation of keratinocytes [8-10].

Within the reporting specialist dental clinic the standard physiologic saline with low dose aqueous chlorine was used from 2000 to 2014 in addition to the more commonly reported approach of no socket irrigation and rinse with saline and or chlorhexidine mouth-rinse. Because it was perceived that outcomes following dental extraction surgery had improved a full retrospective audit of clinical outcomes was performed. The primary objective of the retrospective audit was to record outcomes and, in particular, incidence of alveolar osteitis following surgical dental extraction. The audit was performed via analysis of data reporting consecutive dental extraction surgeries.

\section{Methods}

This audit was designed to compare the outcomes of the two, standard, wound-care treatments used in the clinic over the period examined; where treatment of surgical sites was either no socket irrigation and saline mouth rinse or irrigation of the dental extraction socket with low dose aqueous chlorine in physiological saline. The study was carried out as a consequence of the observation that outcomes seemed to be improved when the dental extraction sites were irrigated with physiologic saline with low dose aqueous chlorine and the same composition used as a mouth rinse. The study was conducted at a dental practise in Melbourne Cambridgeshire between July 2000 and May 2014 and was carried out in accordance with the ICH Guidance for Industry E6 Good Clinical Practice (ICH-GCP) and the Declaration of Helsinki (52nd WMA General Assembly, Edinburgh, October 2000, with clarification on Paragraph 29 by 53rd WMA General Assembly, Washington, October 2000) and conducted by scientifically and medically qualified persons. As per standard procedure, all patients consented to dental extraction.

The audit was of consecutive dental extractions within the specialist dental practice where the dental extraction was performed by one of the two clinicians. Both clinicians had similar levels of training, experience and competence in performing the oral surgery procedures. No pre- or post-operative antibiotics were prescribed unless indicated at the post-surgical review. The individuals represented a normal cross-section of this patient population and included people with concomitant co-morbidities including diabetes, post-splenectomy, hypertension and coronary heart disease. No patients who were fit 
enough for a dental extraction under local anaesthetic where excluded. Only patients requiring general anaesthesia were excluded.

Patients attended via routine, out-patient appointment following a consultation. They had not been starved and had taken any routine medications as prescribed by their general medical practitioner. Anaesthesia / analgesia was achieved via standard approaches using standard local anaesthetic solutions licensed in the UK (lignocaine hydrochloride $2 \%$ with 1 in 80,000 adrenaline) and the dental extractions were carried out using standard techniques. Haemostasis was achieved via local pressure over the socket with cotton wool rolls and biting pressure. Occasionally, 3/0 braided silk sutures were required.

Within the physiologic saline \& aqueous chlorine group, once the tooth was extracted the area was irrigated with aqueous chlorine $(9.6 \mathrm{mM} / 500 \mathrm{ppm})$ in physiological saline as a wash using up to $10-20 \mathrm{ml}$ of the solution in $3 \mathrm{ml}$ aliquots. Dental cotton rolls were soaked in the same solution and the patient applied biting pressure onto the wound via the soaked cotton rolls. Home-care instructions were given that the patient should rinse four times a day with $10 \mathrm{mls}$ of this solution for five days.

Within the physiologic saline group all protocols were identical except the wound was not irrigated at all and the cotton rolls were either dry or soaked in saline solution. Home-care instructions were given that instructed the patient to rinse four times a day with warm salt-water mouth-rinse or $0.2 \%$ chlorhexidine anti-microbial solution (Corsodyl - GSK UK) mouth-rinse for five days. The post-surgery rinse protocol is currently the most frequently recommended standard treatment.

Once the procedure was completed and haemostasis achieved the patient was discharged home with care instructions. All of the study group were reviewed a week after the surgical procedure to assess pain and the success of healing. Additionally, if a patient returned within 14 days with increasing pain and/or un-successful healing and/or infection it was classified as alveolar osteitis.

\section{Statistical Plan}

The audit collated the data sets to test for a difference in the mean proportion of alveolar osteitis between the two groups. The audit analysis powered for superiority of an absolute difference with a power of $95 \%$ and a type I error rate of $5 \%$. Estimation of the rate of alveolar osteitis has been reported as $30 \%$ in the literature and as this was in keeping with clinical experience this was the expected rate under the null hypothesis. A superiority margin of $5 \%$ with a minimum effect of interest of $5 \%$ were selected as being likely to be a clinically meaningful improvement. From this we calculated the sample size to be 934 extractions and planned analyses were scheduled for each third of the trial. In April 2009 with extraction numbers running at 206 (treatment arm) and 106 (control), interim data analyses revealed a large beneficial clinical effect in the treatment arm. The control arm of the study was suspended, and a singlearm extension study continued to more fully assess the benefits and identify any potential adverse effects. The treatment arm continued recruiting until a total of 401 extractions were made. 
The odds ratio and relative risk were calculated from the results using the online calculator at https://www.medcalc.org. The data was also analysed using the T test in Excel.

\section{Results}

Since some patients had multiple extractions, the data was analysed both on a per extraction outcome and on a per person outcome and this is shown in Table 1. It's clear from this Table that treatment with aqueous chlorine in physiological saline had a dramatic effect on the incidence of alveolar osteitis with a significant reduction in the incidence of the condition in those receiving the treatment compared with controls.

Whilst treatment reduced the incidence by a factor of 10 between groups, analysis of the incidence of alveolar osteitis per extraction in the treated and control groups revealed an odds ratio of 0.0191 and analysed per person revealed an odds ratio of 0.0146 . Both analyses showed a clear and significant association between exposure to the treatment regimen and the subsequent outcome $(P<0.0001)$. The relative risk was calculated per patient at $0.029(95 \% \mathrm{Cl} 0.0093$ to 0.0928$)$ showing the results were statistically significant $(p<0.001)$

Whilst there was a slight difference in the ratio of males to females in the treated group compared to the control group is seems unlikely that this difference could account for the dramatic difference in the incidence of the condition. Previous studies have linked infection with alveolar osteitis and so we noted the incidence of pre-operative infection which we considered could be a confounding variable in the results. However, our treatment group had a higher pre-operative rate of infection than the control group and so this could be discounted as a contributing factor to the outcome.

\section{Discussion}

Alveolar osteitis is a common, painful and debilitating condition where multiple forms of treatment have been tested to alleviate the condition, without success. This is the first study to show a clear statistically significant reduction in the incidence of alveolar osteitis following surgical dental extraction using a low dose solution of aqueous chlorine in physiological saline. Use of low dose aqueous chlorine in physiologic saline protocols have been reported as useful in the treatment of burn sites, where systemic antibiotic use has limitations due to the damage to the capillary bed and resultant poor perfusion $[12,13]$.

A recent systematic review revealed that bacteria may play an important role in the pathogenesis of this condition [11] and whilst the use of antibiotics have been suggested to be used postoperatively concerns have arisen because antimicrobial resistance is universally understood to represent a major threat to the provision of healthcare and, in particular, to the risk of infection following elective surgery.

Antibiotic stewardship requires a reduction in the use of antibiotics and places a responsibility on the clinician to use alternative strategies for preventing surgical site infection. Our treatment using aqueous chlorine in physiological saline used as a routine pharmaceutical wound wash in this series of oral 
surgical procedures resulted in a significant improvement in healing outcomes. Furthermore, this was seen in the absence of any use of antibiotics, which is of great potential value in the development of responsible antibiotic stewardship.

An unexpected benefit was consistently seen in the physiologic saline and aqueous chlorine treated group, where patients reported perceiving no pain or very little pain and furthermore, several patients reported that if any soreness developed, aqueous chlorine in physiological saline as a mouth-rinse eliminated it.

If the results of this study can be replicated in other surgical sites and in prospective use, the novel proprietary aqueous chlorine in physiological saline has great potential for use across a wide range of elective and emergency surgical sites to improve clinical outcomes and antibiotic stewardship.

\section{Conclusion}

Aqueous chlorine in physiological saline when used at a low concentration has a significant effect on reducing alveolar osteitis in patients following dental extraction. This is important for two reasons; first, alveolar osteitis is a painful condition which has previous seemed intractable to treatment and second, this has been achieved without the prophylactic use of antibiotics which has great potential to improve clinical outcomes and antibiotic stewardship.

\section{Declarations}

\section{Ethics approval and consent to participate}

Not applicable / waived.

The audit reports on treatment outcomes following routine dental extraction surgery that had been carried out at a dental practise in Melbourne Cambridgeshire between July 2000 and May 2014 using one of two standard approaches to dental extraction surgical site management. The study was designed as an audit of treatments already in use and advised on by Professor R Aspinall (translational medicine) on basis that the treatments were existing treatments and already being used within the clinic.

\section{Consent for publication}

Not applicable

\section{Availability of data and materials}

The datasets generated during the current study are not currently publicly available but are available from the corresponding author on reasonable request. 


\section{Competing interests}

MD, RA TK hold shares or share options in Hypo-stream Ltd. The study pre-dated the formation of HypoStream Ltd.

\section{Funding}

Clinical materials and time for the audit was funded by Hypo-Stream Ltd 31A Charnham Street, Hungerford, Berkshire RG17 0EJ

\section{Authors' contributions}

MD, RA and TK designed the study, MD RA and TK analysed the data and wrote the manuscript. All authors have read and approved the manuscript.

\section{Acknowledgements}

We would like to thank all those who participated in this study including Mr Dominic Flannagan, Private Dental Practice Cambridge CB4 2AE.

We would like to thank the following for advice on nosocomial infections and impact on wider surgical specialities as well as healthcare providers as well as antibiotic stewardship:

Mr Nigel Hall. General Surgery Department, Cambridge University Hospital Addenbrookes, Cambridge CB2 OQQ

Dr Nicholas Brown. Microbiology, Cambridge University Hospital, Addenbrookes, Cambridge CB2 OQQ

\section{References}

1. Sancho-Puchades M, Herráez-Vilas JM, Berini-Aytés L, Gay-Escoda C: Antibiotic prophylaxis to prevent local infection in Oral Surgery: use or abuse? Med Oral Patol Oral Cir Bucal 2009, 14(1):E2833.

2. Lodi G, Figini L, Sardella A, Carrassi A, Del Fabbro M, Furness S: Antibiotics to prevent complications following tooth extractions. Cochrane Database Syst Rev 2012, 11:CD003811.

3. MacGregor AJ: Aetiology of dry socket: a clinical investigation. Br J Oral Surg 1968, 6(1):49-58.

4. Tolstunov L: Influence of immediate postextraction socket irrigation on development of alveolar osteitis after mandibular third molar removal: a prospective split-mouth study, preliminary report. $\mathrm{Br}$ Dent J 2012, 213(12):597-601. 
5. Yengopal V, Mickenautsch S: Chlorhexidine for the prevention of alveolar osteitis. Int J Oral Maxillofac Surg 2012, 41(10):1253-1264.

6. Ozveri Koyuncu B, Isik G, Ozden Yuce M, Gunbay S, Gunbay T: Effect of concentrated growth factors on frequency of alveolar Osteitis following partially-erupted mandibular third molar surgery: a randomized controlled clinical study. BMC Oral Health 2020, 20(1):222.

7. Sakarya S, Gunay N, Karakulak M, Ozturk B, Ertugrul B: Hypochlorous Acid: an ideal wound care agent with powerful microbicidal, antibiofilm, and wound healing potency. Wounds 2014, 26(12):342-350.

8. Leung TH, Zhang LF, Wang J, Ning S, Knox SJ, Kim SK: Topical hypochlorite ameliorates NF-KBmediated skin diseases in mice. J Clin Invest 2013, 123(12):5361-5370.

9. Thomas GW, Rael LT, Bar-Or R, Shimonkevitz R, Mains CW, Slone DS, Craun ML, Bar-Or D: Mechanisms of delayed wound healing by commonly used antiseptics. J Trauma 2009, 66(1):8290; discussion 90-81.

10. Winter J, Ilbert M, Graf PC, Ozcelik D, Jakob U: Bleach activates a redox-regulated chaperone by oxidative protein unfolding. Cell 2008, 135(4):691-701.

11. Riba-Teres N, Jorba-Garcia A, Toledano-Serrabona J, Aguilar-Duran L, Figueiredo R, ValmasedaCastellon E: Microbiota of alveolar osteitis after permanent tooth extractions: A systematic review. $J$ Stomatol Oral Maxillofac Surg 2021, 122(2):173-181.

12. Heggers JA, Sazy BD, Stenberg LL, Strock RL, McCauley DN, Herndon DN, Robson M.C: Bactericidal and wound healing properties of sodium hypochlorite solutions: Journal of Burn Care Rehabilitation 1991,$12 ; 265-268$

13. Hirshowitz B, Moscona AR, Lefler E: Milton for the treatment of burns using the irrigation technique. Burns 1978,5;282-284.

14. Coetzee $E$, Whitelaw $A$, Khan $D$, Rode $H$ : The use of un-buffered sodium hypochlorite in the management of burn wound infection. Burns 2012, 38;529-533

15. Panasenko OM, Gorudko IV, Sokolov AV: Hypochlorous acid as a precursor of free radicals in living systems. Biochemistry (Moscow) 2013, 78; 1466-1489

16. Hidalgo $\mathrm{H}$, Bartolome R, Dominguez $\mathrm{C}$ : Cytotoxicity mechanisms of sodium hypochlorite in cultured human dermal fibroblasts and its bactericidal effectiveness. Chemico-Biological Interactions, 139; 265-282.

17. Klebanoff SJ: Myeloperoxidase: Friend and foe. Journal of Leukocyte Biology 2005, 77 (5); 598-625

\section{Tables}

Table 1 Study Subjects by gender and outcome following surgery

Study Subjects 


\begin{tabular}{|lll|}
\hline Characteristic & Control group & Treated group \\
\hline Number of extractions & 106 & 401 \\
\hline Number of patients & 59 & 201 \\
\hline Male:Female ratio & 1.18 & 0.64 \\
\hline Cases of Alveolar Osteitis & 30 & 3 \\
\hline Incidence (\%) Alveolar Osteitis per extraction & $28.3 \%$ & $0.75 \% *$ \\
\hline
\end{tabular}

* indicates significant difference where $p<0.01$ 